\title{
Extracellular microRNAs initiate immunostimulation via activating toll-like receptor signaling pathways
}

\author{
Hongwei Liang, Koby Kidder and Yuan Liu*
}

\begin{abstract}
Since the discovery of the stability of extracellular microRNAs (miRNAs) in plasma and other body fluids about a decade ago, an increasing body of literature has addressed the function of extracellular miRNAs as novel regulators of gene expression. Although many of these studies have demonstrated that extracellular miRNAs modulate the target genes of recipient cells in a conventional base-pairing manner after exosome-mediated secretion and uptake of miRNAs, recent studies have shown that extracellular miRNAs can also play an unconventional role by rapidly modulating innate immunity and neuron excitation via directly binding to Toll-like receptors (TLRs). In this review, we will summarize the literature incremented from studying the direct activation of TLR signaling pathways by miRNAs and miRNA-like fragments in modulating immune responses.
\end{abstract}

Keywords: Extracellular microRNAs, Immunostimulation, Toll-like receptor

\section{Introduction}

The conventional working model for miRNAs, a class of naturally occurring small noncoding RNAs of 19-24 nt in length, is to function via base-pairing with complementary sites on target mRNAs, causing either translational repression or direct mRNA degradation $[1,2]$. For extracellular miRNAs, it is also generally accepted that they are taken up by recipient cells wherein they behave as endogenous miRNAs to modulate target gene expression through the base-pairing mechanism [1-6]. However, recent studies suggest that extracellular miRNAs may work in a more sophisticated manner [7-9]. For example, extracellular miRNAs, regardless of outside or inside the recipient cells, can serve as physiological ligands for Toll-like receptor 7 (TLR7, mouse) or 8 (TLR8, human), initiating dendritic cell immune responses [7] and spread of central nerve system (CNS) damage [8]. Given that this function of extracellular miRNAs is independent of their conventional role in post-transcriptional gene regulation, it reveals an intriguing and unusual working model of extracellular miRNAs. Herein, we describe the

\footnotetext{
* Correspondence: yliu@gsu.edu

From Center for Inflammation, Immunity and Infection \& Program of Cellular Biology and Immunology, Department of Biology, Georgia State University, Atlanta, GA 30303, USA
}

latest insights in the binding and activation of intracellular TLRs by extracellular miRNAs.

\section{Recognition of specific miRNAs by intracellular TLR7 and TLR8}

Innate immune cells play a critical role in host defense against invading pathogens, including microbial components and mitochondrial DNA fragments derived from apoptotic cells $[10,11]$. To fulfill the function of detecting broad pathogen-associated patterns and danger-associated patterns, these innate immune cells express pattern recognition receptors (PRRs). TLRs are one of the most studied families of PRRs and their activation promotes both innate inflammatory responses and the induction of adaptive immunity [12]. Due to their broad and complicated function in innate immunity, TLRs may be considered a 'Swiss Army knife' of the immune system -replete with multifaceted responses for various infectious and disease states [12]. There are multiple TLRs expressed in immune cells, and of these, TLR1, TLR2, TLR4, TLR5 and TLR6 are expressed primarily on the cell surface where they recognize lipopolysaccharides or other unique molecules derived from microbes $[10,11]$. As opposed to these cell surface TLRs, innate immune cells also express intracellular TLRs (i.e. TLR3, TLR7, TLR8 and TLR9)which are 
mainly localized at the membranes of endolysosomal compartments and can trigger the induction of cytokines essential for innate immune responses [10, 11, 13]. For example, Ramirez-Ortiz et al. have demonstrated that TLR7 and the adaptor molecule Myd88 can be recruited to the endolysosomal compartment by the receptor TREML4, which subsequently amplifies TLR7-induced type I interferon responses [13]. Both cell surface and intracellular TLRsare intrinsically capable of detecting foreign nucleic acids, including double-stranded RNA (dsRNA) and single-stranded RNA (ssRNA) of RNA viruses and DNA from bacteria and DNA viruses [10, $11,14,15]$. Furthermore, a previous study by Heilet al. demonstrated that murine TLR7 or human TLR8 can recognize GU-rich ssRNA derived from human immunodeficiency virus-1 (HIV-1) [16]. Kariko et al. [14] found that small interfering RNAs (siRNAs) mediate sequence-independent gene suppression and induce immune activation by signaling through TLR3. In line with this, Kleinman et al. [15] showed that generic siRNAs may suppress choroidal neovascularization $(\mathrm{CNV})$ via interacting with TLR3, thereby inducing interferon-gamma and interleukin-12 production.Therefore, serving as important RNA sensors, intracellular TLRs especially TLR3, TLR7 and TLR8 can detect unique foreign nucleic acids and thus initiate TLR-mediated innate immune responses.

Given that almost all cell types can secrete and deliver extracellular miRNAs to recipient cells via exosomes $[4,5]$, it is possible that secreted miRNAs can reach intracellular TLRs in the recipient cells and activate TLR-mediated signaling pathways. Several recent studies have shown that certain miRNAs can bind to immune cell TLR7 in mice or TLR8 in humans and induce inflammatory cytokine secretion (Table 1) [7, 8, 17-25]. The study by Fabbri and colleagues [17] revealed that tumor-secreted miR-21 and miR-29a were delivered via exosomes into surrounding macrophages where they activated TLR8 (homologous to murine TLR7) to trigger a pro-inflammatory response. This finding suggests that activating the macrophage inflammatory responses by these oncomiRs may facilitate tumor metastasis. In line with this, $\mathrm{He}$ et al. showed that tumor-secreted miR-21 induced myoblast apoptosis in cancer cachexia via TLR7-c-Jun N-terminal kinasedependent pathway [18]. Their study also confirmed that the pro-apoptotic activity of miR-21 is mediated through the binding to and subsequent signaling byTLR7, resulting in apoptosis of murine myoblasts. Lehmann et al. [8] reported that let- 7 could serve as a signaling molecule to directly activate neuronal RNA-sensing TLR7 and cause neurodegeneration. Supporting this anomalous role of let-7 in activating the TLR7 signaling pathway, they found that TLR7-deficient mice were resistant to such neurodegenerative effect, while this susceptibility to let-7 could be restored in neurons transfected with TLR7. In agreement with this, Park et al. [19] found that let-7b induces TLR7/ TRPA1-dependent single-channel activities in neurons and HEK293 cells overexpressing TLR7/TRPA1, and that intraplantar injection of let-7b elicits rapid spontaneous pain via activating TLR7 and TRPA1. Furthermore, their study also showed that the binding of let-7 with TLR7 requires the GUUGUGU motif, a core GU-rich motif that is also present in HIV ssRNA40, a known TLR7 ligand [16]. In fact, a GU-rich motif was identified in all TLR7/8-binding miRNA (GUUG for miR-21, GGUU for miR-29a and GUUGUGU for let-7b). The recognition of $U$ and UG-rich motifs on miRNA is consistent with the involvement of TLR7 and TLR8 [26, 27], whose activation is sequence-specific $[28,29]$.

Table 1 Extracellular miRNAs serve as the ligand for TLR7/8

\begin{tabular}{|c|c|c|c|c|}
\hline miRNA & Source & Receipt cells & Cytokine & Ref \\
\hline $\begin{array}{l}\text { miR-34a, }-122,-133 a,-142,-146 a, \\
\text { and }-208 a\end{array}$ & plasma & macrophages and cardiomyocytes & MIP-2, TNF-a, L-6 & Chao W et al [20] \\
\hline miR-29a & serum & Dendritic Cells & TNF-a, IL-6 & Garzon R et al [7] \\
\hline miR-let-7b & $\begin{array}{l}\text { Rheumatoid Arthritis Synovial } \\
\text { Fluid }\end{array}$ & macrophage & $\begin{array}{l}\text { TNF-a, } \\
\text { IL-8, IL-7 }\end{array}$ & Shahrara $\mathrm{S}$ et al [21] \\
\hline $\operatorname{miR}-21$ & $\begin{array}{l}\text { extracellular vesicles from the } \\
\text { brains of rhesus macaques }\end{array}$ & macrophage/microglial cells & IL-6, TNF-a & Fox HS et al [22] \\
\hline miR-let-7c and - 21 & $\begin{array}{l}\text { exosomes of the neuronal cultures } \\
\text { and developing brains }\end{array}$ & neurons & & Hsueh YP et al [23] \\
\hline miR-29b & exosomes from beta-cells & spleen cells & TNF-a & Bach JM et al [24] \\
\hline miRNA-let-7b & dorsal root ganglion neurons & dorsal root ganglion neurons & & Ji RR et al [19] \\
\hline miR-21 & $\begin{array}{l}\text { lung cancer- and pancreatic } \\
\text { cancer-derived microvesicles }\end{array}$ & myoblasts & & Croce CM et al [18] \\
\hline MicroRNA-146a & plasmacytoid dendritic cells & plasmacytoid dendritic cells & & Blom B et al [25] \\
\hline miR-21, -147 , and $-29 a$ & exosomes of cancer cells & macrophages & TNF-a, IL-6 & Croce CM et al [17] \\
\hline miR-let-7 & Cerebrospinal fluid & macrophage/microglial cells & TNF- $a$ & Lehnardt $\mathrm{S}$ et al [8] \\
\hline
\end{tabular}


By specifically delivering let-7b into tumor-associated macrophages (TAMs), Huang et al. [30] also showed that let-7b efficiently reprogrammed the functions of TAMs, reversing the suppressive tumor microenvironment and inhibiting tumor growth. Given that this GU-rich motif is shared by various miRNAs, it is highly possible that intracellular TLR7 or TLR8 can be targeted by these miRNAs under different physiological and pathophysiological settings. Earlier work by Judge et al. [31] showed that GU-rich motifs contributed to immune stimulation through interacting with intracellular TLR. In a similar fashion, miR-122, a predominant miRNA found in the liver, also contains two occurrences of an alternating U/G motif, and is associated with immune stimulation. Furthermore, modifying the U/G motif significantly reduces the immune stimulatory effect of endogenous miR-122 or miR-122-mimetic RNA [32].

\section{Direct binding to immune cell TLRs by non-coding small RNAs from other species}

Following the discovery of cross-kingdom regulation mediated by plant miRNAs in mammalian tissues and cells $[3,33]$, an increasing body of literature has shown that exogenous miRNAs derived from various species, including plants and viruses, play a critical role in modulating mammalian cell function [34-36]. Although these exogenous miRNAs execute their biological function in the recipient cells mainly through base-pairing the transcript of target genes in a manner of endogenous miRNA [34, 35], certain exogenous miRNAs that contain the TLR-binding GU-rich motif can also directly bind to intracellular TLRs or cell surfaces, leading to activation of TLR-mediated immune responses. In fact, given that the levels of exogenous miRNAs inside human and animals are extremely low, these exogenous miRNAs may have difficulty to be recruited by RNA-induced silencing complex (RISC)-loading complex in recipient cells. However, through the unorthodox way of directly binding to TLRs, exogenous miRNAs can elicit rapid biological responses in human and murine cells at extremely low concentration. Indeed, Cavalieri et al. [37] found that plant miRNAs bound to TLR3 in dendritic cells, thereby impairing TRIF signaling, limited $\mathrm{T}$ cell proliferation and dampened dendritic cell immune responses. Interestingly, their studies further showed that the anti-inflammatory efficacy was associated with various miRNAs derived from different plants, and that the immuno-modulatory effect of plant miRNA was independent of sequence or plant type. A

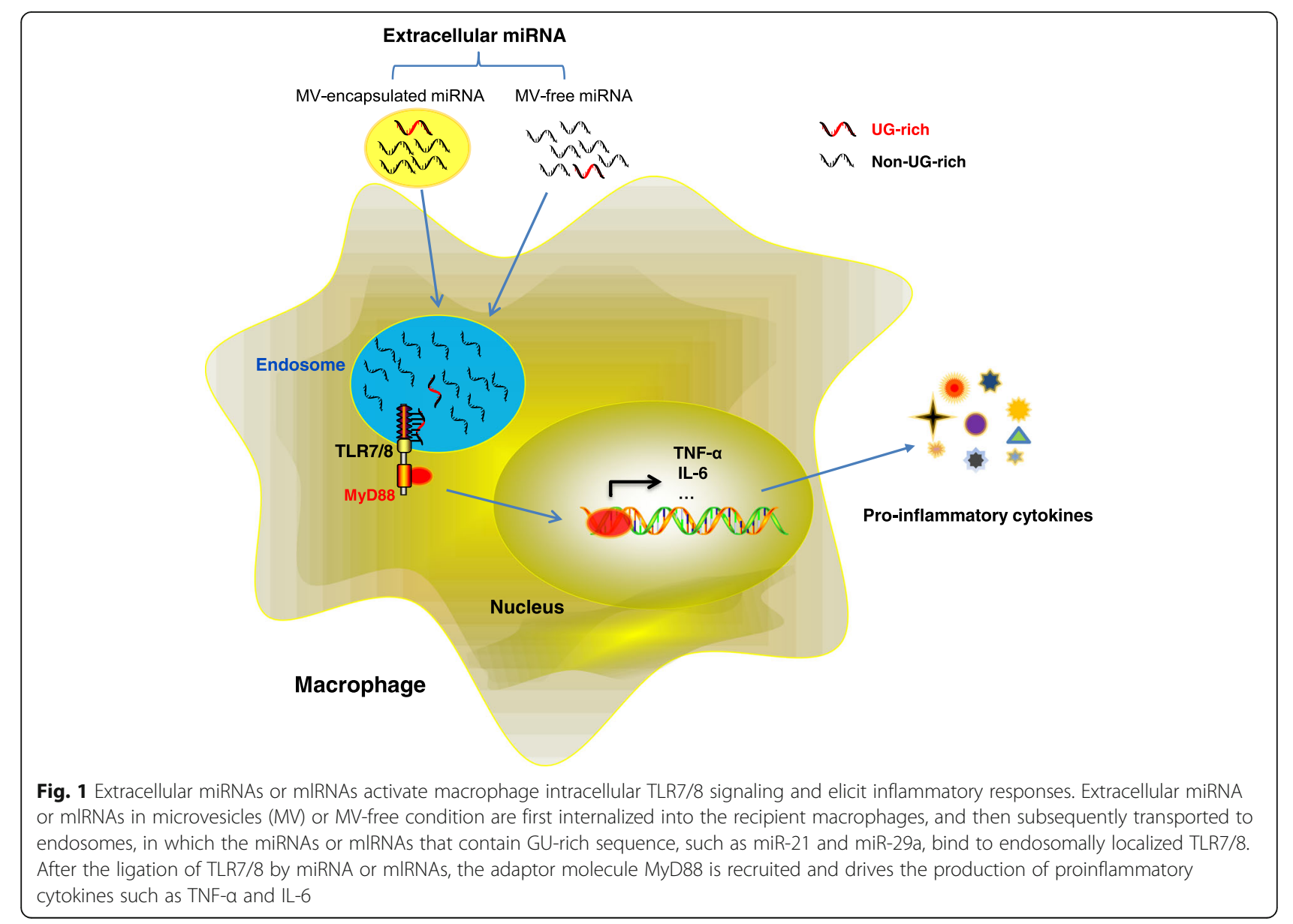


previous study by Sampey and colleagues also suggested that exosomes containing trans-activating response (TAR) element RNA enhanced susceptibility of undifferentiated naive cells to HIV-1 infection [38]. TAR was found to be able to bind to TLR3, while the $5^{\prime}$ and $3^{\prime}$ stems (TAR miRNAs) bound best to TLR7 and 8, respectively. Through binding to TLRs, TAR miRNA can activate the NF- $\mathrm{kB}$ pathway and regulate cytokine expression and secretion. This finding provides a novel mechanism underlying the inflammation observed in HIV-1-infected patients undergoing combination antiretroviral therapy (cART) [38].

It has been generally accepted that bacteria alone cannot produce miRNAs; however, a recent study by $\mathrm{Gu}$ et al. [39] demonstrated that Salmonella, a leading cause of food-borne illness worldwide, can not only release viral non-coding RNA fragments into the infected host cells, but also hijack the host cell non-classical miRNA processing machinery to further process these viral non-coding RNAs into 22-nt functional RNA fragments. Given that the bacterial 'miRNA-like' fragments generated in the host cells may also possess GU or UG-rich motifs, these bacterial 'miRNA-like' fragments can also elicit host cell inflammatory responses through binding to intracellular TLRs and activating TLR-mediated signal pathways.

\section{Conclusion}

In summary, extracellular miRNAs or miRNA-like small RNA fragments (mlRNAs) can regulate recipient cell function through both conventional and unconventional ways. Conventionally, miRNAs or mIRNAs repress protein expression at the post-transcriptional level through a base-pairing mechanism; whereas unconventionally, miRNAs or mlRNAs directly serve as ligands of TLRs. The working model of miRNAs or mIRNAs serving as TLR ligands is illustrated in Fig. 1. Extracellular miRNA or mlRNAs under microvesicles (MV) or MV-free conditions are first internalized into the recipient macrophages, and then subsequently transported to endosomes, in which the miRNAs or mlRNAs that contain the GU-rich sequence, such as miR-21 and miR-29a, bind to endosomal TLR7/8. After GU-enriched miRNA or mIRNAs sensed by TLR7/8, the adaptor molecule MyD88 is recruited to drive the production of proinflammatory cytokines including TNF- $\alpha$, IL- 6 and IFN $\gamma$. Although many fundamental issues remain to be further addressed, the discovery of miRNAs or mIRNAs directly serving as TLR7/8 ligands in immune cells significantly expands the field of miRNA research, and provides potentially novel therapeutic targets in controlling innate immune response and inflammation.

\section{Abbreviations}

CART: Combination antiretroviral therapy; CNS: Central nerve system; dsRNA: Double-stranded RNA; HIV-1: Human immunodeficiency virus-1; miRNAs: microRNAs: mIRNAs: miRNA-like small RNA fragments: MV: Microvesicles; PRRs: Pattern recognition receptors; RISC: RNA-induced silencing complex; siRNAs: Small interfering RNAs; ssRNA: Single-stranded RNA; TAR: Trans-activating response; TLRs: Toll-like receptors

Acknowledgements

Not applicable.

\section{Funding}

This work was supported, in part, by grants from National Institutes of Health (Al106839) and the American Cancer Society (to Y.L.), and the China

Postdoctoral Science Foundation (2014 M550284).

Availability of data and materials

Not applicable.

\section{Authors' contributions}

$\mathrm{HL}$ and $\mathrm{KK}$ searched the literature, and $\mathrm{YL}$ wrote the manuscript. All authors read and approved the final manuscript.

Ethics approval and consent to participate

Not applicable.

\section{Consent for publication}

Not applicable.

\section{Competing interests}

The authors declare that they have no competing interests.

\section{Publisher's Note}

Springer Nature remains neutral with regard to jurisdictional claims in published maps and institutional affiliations.

Received: 18 December 2017 Accepted: 23 January 2019

Published online: 14 February 2019

\section{References}

1. Bartel DP. MicroRNAs: genomics, biogenesis, mechanism, and function. Cell. 2004;116:281-97.

2. Ambros $V$. The functions of animal microRNAs. Nature. 2004:431:350-5.

3. Zhang $L$, et al. Exogenous plant MIR168a specifically targets mammalian LDLRAP1: evidence of cross-kingdom regulation by microRNA. Cell Res. 2012:22:107-26

4. Zhang $Y$, et al. Microvesicle-mediated delivery of transforming growth factor beta1 siRNA for the suppression of tumor growth in mice. Biomaterials. 2014;35:4390-400

5. Zhang $Y$, et al. Secreted monocytic miR-150 enhances targeted endothelial cell migration. Mol Cell. 2010;39:133-44.

6. Chen $X$, Liang $H$, Zhang J, Zen K, Zhang CY. Secreted microRNAs: a new form of intercellular communication. Trends Cell Biol. 2012;22:125-32.

7. Ranganathan $\mathrm{P}$, et al. Serum miR-29a is upregulated in acute graft-versushost disease and activates dendritic cells through TLR binding. J Immunol. 2017;198:2500-12.

8. Lehmann SM, et al. An unconventional role for miRNA: let-7 activates toll-like receptor 7 and causes neurodegeneration. Nat Neurosci. 2012;15:827-35.

9. Sarvestani ST, et al. Sequence-dependent off-target inhibition of TLR7/8 sensing by synthetic microRNA inhibitors. Nucleic Acids Res. 2015;43:1177-88.

10. Takeda K, Kaisho T, Akira S. Toll-like receptors. Annu Rev Immunol. 2003:21: 335-76

11. Blasius AL, et al. Slc15a4, AP-3, and Hermansky-Pudlak syndrome proteins are required for toll-like receptor signaling in plasmacytoid dendritic cells. Proc Natl Acad Sci U S A. 2010;107:19973-8.

12. Dowling JK, Mansell A. Toll-like receptors: the swiss army knife of immunity and vaccine development. Clin Transl Immunology. 2016;5:e85

13. Ramirez-Ortiz ZG, et al. The receptor TREML4 amplifies TLR7-mediated signaling during antiviral responses and autoimmunity. Nat Immunol. 2015; 16:495-504

14. Kariko K, Bhuyan P, Capodici J, Weissman D. Small interfering RNAs mediate sequence-independent gene suppression and induce immune activation by signaling through toll-like receptor 3. J Immunol. 2004;172:6545-9.

15. Kleinman ME, et al. Sequence- and target-independent angiogenesis suppression by siRNA via TLR3. Nature. 2008;452:591-7. 
16. Heil F, et al. Species-specific recognition of single-stranded RNA via toll-like receptor 7 and 8. Science. 2004;303:1526-9.

17. Fabbri M, et al. MicroRNAs bind to toll-like receptors to induce prometastatic inflammatory response. Proc Natl Acad Sci U S A. 2012;109: E2110-6.

18. He WA, et al. Microvesicles containing miRNAs promote muscle cell death in cancer cachexia via TLR7. Proc Natl Acad Sci U S A. 2014;111:4525-9.

19. Park CK, et al. Extracellular microRNAs activate nociceptor neurons to elicit pain via TLR7 and TRPA1. Neuron. 2014;82:47-54.

20. Feng $Y$, et al. Extracellular MicroRNAs induce potent innate immune responses via TLR7/MyD88-dependent mechanisms. J Immunol. 2017; 199:2106-17.

21. Kim SJ, et al. Identification of a novel toll-like receptor 7 endogenous ligand in rheumatoid arthritis synovial fluid that can provoke arthritic joint inflammation. Arthritis Rheumatol. 2016:68:1099-110.

22. Yelamanchili SV, et al. MiR-21 in extracellular vesicles leads to neurotoxicity via TLR7 signaling in SIV neurological disease. PLoS Pathog. 2015;11:e1005032.

23. Liu HY, Huang CM, Hung YF, Hsueh YP. The microRNAs Let7c and miR2 are recognized by neuronal toll-like receptor 7 to restrict dendritic growth of neurons. Exp Neurol. 2015;269:202-12.

24. Salama A, et al. MicroRNA-29b modulates innate and antigen-specific immune responses in mouse models of autoimmunity. PLoS One. 2014; 9:e106153.

25. Karrich JJ, et al. MicroRNA-146a regulates survival and maturation of human plasmacytoid dendritic cells. Blood. 2013;122:3001-9.

26. Hornung $V$, et al. Sequence-specific potent induction of IFN-alpha by short interfering RNA in plasmacytoid dendritic cells through TLR7. Nat Med. 2005;11:263-70.

27. Sioud M. Induction of inflammatory cytokines and interferon responses by double-stranded and single-stranded siRNAs is sequence-dependent and requires endosomal localization. J Mol Biol. 2005;348:1079-90.

28. Forsbach A, et al. Identification of RNA sequence motifs stimulating sequence-specific TLR8-dependent immune responses. J Immunol. 2008; 180:3729-38.

29. Diebold SS, et al. Nucleic acid agonists for toll-like receptor 7 are defined by the presence of uridine ribonucleotides. Eur J Immunol. 2006;36:3256-67.

30. Huang $Z$, et al. Targeted delivery of let-7b to reprogramme tumorassociated macrophages and tumor infiltrating dendritic cells for tumor rejection. Biomaterials. 2016;90:72-84.

31. Judge $A D$, et al. Sequence-dependent stimulation of the mammalian innate immune response by synthetic siRNA. Nat Biotechnol. 2005;23:457-62.

32. Peacock $\mathrm{H}$, et al. Nucleobase and ribose modifications control immunostimulation by a microRNA-122-mimetic RNA. J Am Chem Soc. 2011:133:9200-3.

33. Zhou Z, et al. Honeysuckle-encoded atypical microRNA2911 directly targets influenza a viruses. Cell Res. 2015;25:39-49.

34. Landais I, et al. Human cytomegalovirus miR-UL112-3p targets TLR2 and modulates the TLR2/IRAK1/NFkappaB signaling pathway. PLoS Pathog. 2015; 11:e1004881.

35. Chen $Y$, et al. HCV-induced miR-21 contributes to evasion of host immune system by targeting MyD88 and IRAK1. PLoS Pathog. 2013;9:e1003248.

36. Lukasik A, Zielenkiewicz P. Plant MicroRNAs-novel players in natural medicine? Int J Mol Sci. 2016;18(1).

37. Cavalieri $\mathrm{D}$, et al. Plant microRNAs as novel immunomodulatory agents. Sci Rep. 2016;6:25761.

38. Sampey GC, et al. Exosomes from HIV-1-infected cells stimulate production of pro-inflammatory cytokines through trans-activating response (TAR) RNA. J Biol Chem. 2016;291:1251-66.

39. Gu H, et al. Salmonella produce microRNA-like RNA fragment Sal-1 in the infected cells to facilitate intracellular survival. Sci Rep. 2017;7:2392.

Ready to submit your research? Choose BMC and benefit from:

- fast, convenient online submission

- thorough peer review by experienced researchers in your field

- rapid publication on acceptance

- support for research data, including large and complex data types

- gold Open Access which fosters wider collaboration and increased citations

- maximum visibility for your research: over $100 \mathrm{M}$ website views per year

At $\mathrm{BMC}$, research is always in progress.

Learn more biomedcentral.com/submissions 